\title{
Clustering of galaxies around gamma-ray burst sight-lines ${ }^{\star}$
}

\author{
V. Sudilovsky ${ }^{1}$, J. Greiner ${ }^{1}$, A. Rau ${ }^{1}$, M. Salvato ${ }^{1}$, S. Savaglio ${ }^{1}$, S. D. Vergani ${ }^{2,3}$, P. Schady ${ }^{1}$, J. Elliott ${ }^{1}$, T. Krühler ${ }^{4}$, \\ D. A. Kann ${ }^{5}$, S. Klose ${ }^{5}$, A. Rossi ${ }^{5}$, R. Filgas ${ }^{6}$, and S. Schmidl ${ }^{5}$
}

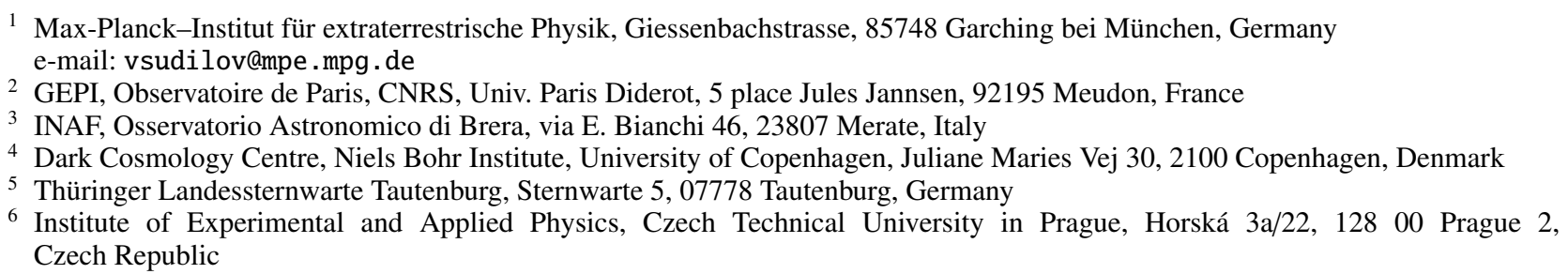

Received 6 February 2013 / Accepted 25 February 2013

\begin{abstract}
There is evidence of an overdensity of strong intervening MgII absorption line systems distributed along the lines of sight toward gamma-ray burst (GRB) afterglows relative to quasar sight-lines. If this excess is real, one should also expect an overdensity of field galaxies around GRB sight-lines, as strong MgII tends to trace these sources. In this work, we test this expectation by calculating the two point angular correlation function of galaxies within $120^{\prime \prime}\left(\sim 470 h_{71}^{-1} \mathrm{Kpc}\right.$ at $\left.\langle z\rangle \sim 0.4\right)$ of GRB afterglows. We compare the gamma-ray burst optical and near-infrared detector (GROND) GRB afterglow sample - one of the largest and most homogeneous samples of GRB fields - with galaxies and active galactic nuclei found in the COSMOS-30 photometric catalog. We find no significant signal of anomalous clustering of galaxies at an estimated median redshift of $z \sim 0.3$ around GRB sight-lines, down to $K_{\mathrm{AB}}<19.3$. This result is contrary to the expectations from the MgII excess derived from GRB afterglow spectroscopy, although many confirmed galaxy counterparts to MgII absorbers may be too faint to detect in our sample - especially those at $z>1$. We note that the addition of higher sensitivity Spitzer/IRAC or HST/WFC3 data for even a subset of our sample would increase this survey's depth by several orders of magnitude, simultaneously increasing statistics and enabling the investigation of a much larger redshift space.
\end{abstract}

Key words. gamma-ray burst: general - galaxies: statistics

\section{Introduction}

Gamma-ray bursts (GRBs) have proven to be powerful tools for studying the high redshift universe. Their afterglows pinpoint star forming galaxies that would otherwise be exceedingly difficult to discover. Using bright transients such as GRBs to study the Universe introduces completely different selection criteria from standard surveying techniques. Long duration GRB (in this work, the term "GRB" implies long-duration, unless explicitly stated otherwise) afterglows have been used to study the star formation and metallicity evolution of the Universe (see e.g. Savaglio et al. 2009; Jakobsson et al. 2005; Prochaska et al. 2006; Salvaterra et al. 2012). The host galaxies of $z>5$ GRB afterglows have been studied by Tanvir et al. (2012) and Basa et al. (2012). The upper limits on the non-detections that the authors derive suggest that the galaxy luminosity function evolves rapidly at these higher redshifts. In general, GRBs seem to be clear tracers of star formation (see e.g. Le Floc'h et al. 2006; Butler et al. 2010), however it is unclear the extent to which selection effects against more dusty and metal-rich galaxies may affect surveys (Krühler et al. 2011; Elliott et al. 2012; Perley et al. 2013).

In addition to studies of the GRB host galaxies, GRB afterglows themselves offer a brief and bright glimpse into the high redshift universe. Intervening absorption line systems can be detected in the same manner employed during the past decades

* Table 1 is available in electronic form at http://www . aanda.org with quasar spectroscopy. One such absorption line system is MgII, which is easily detected in moderate signal-to-noise ratio spectra at $\lambda_{\text {rest }} \sim 2800 \AA$. Because of its strong absorption and ease of identification owing to the fact that it is an absorption doublet, MgII has been used extensively as a tracer of galaxies, galactic outflows, and chemical evolution. MgII itself is coincident with a wide range of neutral hydrogen column densities from $N_{\mathrm{HI}} \sim 10^{16}-10^{22} \mathrm{~cm}^{-2}$ (Churchill et al. 2000), though there is strong evidence that $\mathrm{MgII}$ equivalent width (EW) is directly correlated with higher hydrogen columns and thus smaller impact parameters to the bulk star forming region of galaxies (Steidel et al. 1995; Bouché et al. 2006; Kacprzak \& Churchill 2011; Bordoloi et al. 2011). Indeed, Bordoloi et al. (2012) showed that MgII absorbers follow a bi-modal spatial distribution, wherein at impact parameters smaller than $d \sim 40 \mathrm{Kpc}$, $\mathrm{MgII}$ is associated with cool star forming regions, and at $d>$ $40 \mathrm{Kpc} \mathrm{MgII}$ tends to be uniformly distributed around galaxies, perhaps suggesting large scale outflows. There is no evidence to suggest that this behavior evolves with redshift, at least in the interval $0.4 \leq z \leq 2$. It is, however, worth noting that Matejek et al. (2013) have studied the $2<z<6$ regime using infrared spectra of quasars. The authors find that the association between strong MgII and damped Lyman- $\alpha$ systems (DLAs) strengthens at higher redshifts, even though the overall taxonomy of these absorbers as defined in Churchill et al. (2000) does not change. There is evidence that the redshift evolution in the number density of MgII absorbers follows the cosmic star formation rate (Zhu \& Ménard 2012; Matejek \& Simcoe 2012), though the 
effects of observational bias may still play an important role (López \& Chen 2012).

Prochter et al. (2006) compared the incidence of strong MgII absorbers in quasar sight-lines with those found in GRB afterglows. In this context, "strong" is defined as absorption systems whose rest frame equivalent width of the $\lambda 2796 \AA$ feature is $W_{0} \geq 1.0 \AA$. The authors found a factor of $\sim 4$ excess in the number density of these strong intervening $0.4<z<2.0$ MgII systems in GRB sight-lines relative to those found in quasars sight-lines. More recently, Vergani et al. (2009) have confirmed a factor of $\sim 2$ excess with a much larger sample. Besides increasing the statistical significance of the MgII discrepancy, they found that the properties of weak $0.3 \leq W_{0} \leq$ $1.0 \AA$ intervening MgII systems are statistically identical between the two types of sight-lines. Interestingly, the abundance of CIV - a higher ionization absorption line system - also does not show an overdensity (Sudilovsky et al. 2007; Tejos et al. 2007). Many authors have examined possible solutions to the observed discrepancy, and agree that a satisfactory explanation does not yet exist (Frank et al. 2007; Sudilovsky et al. 2009; Cucchiara et al. 2009; Vergani et al. 2009; Kann et al. 2010; Wyithe et al. 2011; Rapoport et al. 2012). A comprehensive overview of these possible solutions is given in Porciani et al. (2007). It is especially intriguing that the amplitude of the discrepancy seemingly depends on the resolution of the afterglow spectra suggesting either that rapid response high resolution spectroscopy may introduce a not yet understood observational bias in any sample study of GRB afterglows, or that the MgII discrepancy is a statistical fluke (Cucchiara et al. 2012).

Objects near the line of sight to a GRB afterglow are unaffected by light from the event after the afterglow has faded, allowing detailed follow-up of the field to extremely high angular resolutions. The study of sources near quasars are reliant on either extremely high resolution imaging on intrinsically dim quasars, Lyman- $\alpha$ imaging, or very low redshift quasars. Galaxies that give rise to absorption line systems in GRB afterglow spectra have been directly imaged and studied, although the number of robust associations is still extremely small. Chen et al. (2009) find that additional galaxies are found at very close angular distances to GRB host galaxies whose afterglows exhibited strong MgII absorption, though it is unclear what fraction were associated with the absorption line systems. Schulze et al. (2012) proposed galaxy counterpart candidates to absorbers, and additionally found field galaxies with the same redshift as absorption line systems at distances of 130-161 kpc away from the sight-line. The frequency of field galaxies that are associated with $\mathrm{MgII}$ absorption line systems is still unclear. However, Lopez et al. (2008) have shown that strong MgII absorbers are strongly associated with galaxy clusters, and that the number density of strong MgII absorbers around galaxy clusters is much higher than those around field galaxies. In this work, we test for any anomalous signatures of field galaxy clustering in GRB and quasar lines of sight.

If there is indeed a higher probability of detecting strong MgII absorption line systems in GRB afterglow than in quasar sight-lines, one should also expect to detect more galaxies at close angular separations to GRB sight-lines. The two point correlation function is a powerful tool to determine if objects are clustered, and, if so, what their correlation lengths are. The angular two point correlation function is formally defined as

$\mathrm{d} P=n[1+w(\theta)] \mathrm{d} \Omega$,

where $\mathrm{d} P$ is the probability of finding an object within a solid angle $\mathrm{d} \Omega$ at an angular distance $\theta$ (Peebles 1980). In general, a positive $w$ implies some enhancement of object-object grouping above a uniform random distribution, while a negative $w$ implies some avoidance. The two point correlation function has been used to estimate the clustering properties of galaxies, quasars, and GRBs (see e.g. Groth \& Peebles 1977; Ross et al. 2009; Brainerd et al. 1995).

In this work, we measure the angular two-point correlation function between GRB afterglows and field galaxies, and compare this quantity with galaxy-galaxy and AGN-galaxy correlations. In Sect. 2, we present the sample that we analyze in Sect. 3. We present the results of the analysis in Sect. 4, and discuss these results in Sect. 5.

\section{The sample}

The gamma-ray burst optical and near-infrared detector (GROND) is a simultaneous 7-channel imager mounted on the MPG/ESO $2.2 \mathrm{~m}$ telescope at La Silla, Chile (Greiner et al. 2008). The four optical channels are nearly identical to the SDSS $g^{\prime} r^{\prime} i^{\prime} z^{\prime}$ filters, while the infrared channels are effectively equivalent to the 2MASS $J H K$ filters. While the detector system was built to quickly measure photometric redshifts of GRBs via the Lyman- $\alpha$ dropout technique, the multi-wavelength photometry provided by GROND can potentially reveal a wealth of physical characteristics of any source (Greiner et al. 2008).

Our sample of GRB fields is constructed exclusively of GROND observations taken between 2007-2012. This fact ensures a high degree of homogeneity, as no correction for cross-instrumental calibrations are required. Furthermore, the simultaneous nature of GROND observations ensures that data on a per-field basis have not varied due to intrinsic variability or weather conditions. Since only ephemeris, hardware failure and weather losses prevent GROND follow-up observations, the GROND sample of GRB afterglows has an exceptional success rate of $\sim 90 \%$ detection of long-duration GRBs when follow-up is possible within the first few hours after the trigger (Greiner et al. 2011; Krühler et al. 2011). We include in our sample all fields which 1) have a XRT localization of the burst; 2) Galactic latitude $|b|>10^{\circ} ; 3$ ) at least $15^{\circ}$ from the Galactic center; and 4) have been observed to a $3 \sigma$ background limiting magnitude of $K_{\mathrm{AB}} \sim 20.4$. This limiting magnitude corresponds roughly to an integration time of 1500 and $1200 \mathrm{~s}$ in optical and near-infrared (NIR), respectively, among four telescope dither positions. For reference, typical $3 \sigma$ AB limiting magnitudes in the optical are at least 3 mag deeper than $K$, while $J$ and $H$ are 1.0 and 0.5 mag deeper, respectively. Our final sample consists of 73 GRB fields (see Table 1 and Fig. 1). Since most of the bursts have no measured redshift, we assume that our bursts follows the same redshift distribution as in the TOUGH sample (Jakobsson et al. 2012).

\section{Procedure}

\subsection{Creating the source catalog}

To accurately measure the optimal extraction region and measure the apparent extent of sources from GROND multi-band images, we co-add all 7 images to create a single detection image for every field. Since the exposure time differs between the NIR and optical channels, we scale and weigh the images to a common effective exposure. SExtractor (Bertin \& Arnouts 1996) is run on the detection image using relatively low source extraction thresholds such that $5-10 \%$ of the sources are likely spurious detections, but still no bright sources are incorrectly de-blended or 


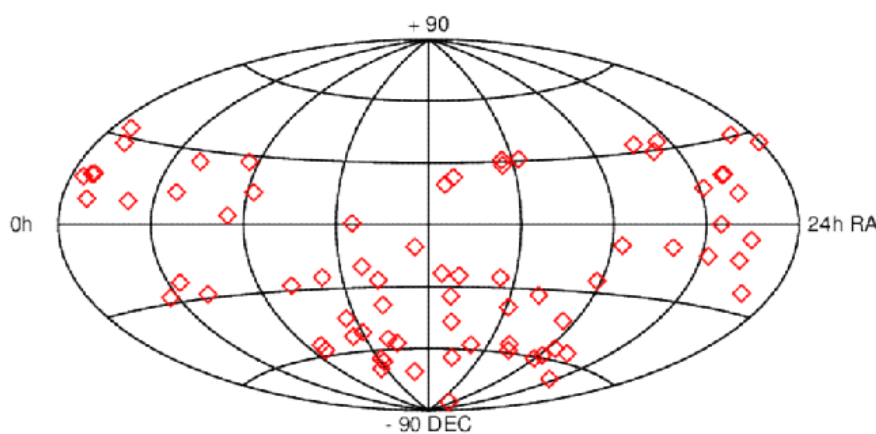

Fig. 1. Positions in equatorial coordinates of the 73 GROND GRB afterglows in the sample. Bursts within $10^{\circ}$ of the Galactic plane are excluded.

otherwise split into multiple sources. The detection image source catalog is correlated with each individual band's source catalog, and only those sources whose 1) positions in each catalog are consistent to within 0.5 of each other; and 2) are detected in at least 6 out of the 7 bands (one of which is required to be a $K$-band detection); and 3) have an error $\sigma_{K} \leq 0.10 \mathrm{mag}$ are used in the final source catalog for that field. The latter criteria limits the overall sample to roughly $\sigma_{K}<19.3$, with $\sim 10 \%$ of sources dimmer than this. The incompleteness down to $K \sim 20.0$ does not introduce any significant bias in the final results. This cross-correlation ensures that spurious detections are removed, and that each source has multi-wavelength detections. The requirement that objects be strongly detected in $K$ ensures that they can be reliably seperated between galaxies and stars.

Creating a source catalog based on a detection image in the aforementioned manner minimizes the probability that flux from extended objects is missed due to varying spectral properties as a function of position. That is, by determining a suitable aperture from the co-added image, one is guaranteed that the entirety of flux in an extended source is included therein if that same aperture is then used in each individual image, as long as that source is strongly detected in each band. Additionally, if the source is reasonably isolated in the image, this method avoids the complications introduced by PSF matching and galaxy fitting while still providing adequate photometry. We therefore perform aperture photometry with SExtractor in dual-mode using the detection image as the template for each band.

The SExtractor magnitudes are calibrated based on timetabulated instrumental zeropoints in the case of the optical channels and 2MASS field stars in the case of the NIR. GROND zeropoints are calibrated on average once every three months based on SDSS standard fields, and immediately after technical work is performed on the optical system. The rms of the optical zeropoints as a function of time is on the order of $0.05 \mathrm{mag}$. We expect a similar spread due to varying atmospheric conditions, as the range of seeing in our sample is between 1.0 and 1.6 in $r^{\prime}$. In 18 fields (24\%) co-incident with the SDSS, magnitudes were calibrated directly against SDSS field stars.

Next, we fit each object's spectral energy distribution (SED) with LePHARE (Arnouts et al. 1999; Ilbert et al. 2006). LePHARE is a spectral template fitting tool based on $\chi^{2}$ minimization. We determine the best fit galaxy and stellar templates for each source, using the COSMOS galaxy templates, with emission lines and prescribed reddening and parameters therein (Ilbert et al. 2009). More specifically, we adopted the Prevot (Prevot et al. 1984) extinction law for late type galaxy templates and the Calzetti law (Calzetti et al. 1994) and two modifications thereof (Ilbert et al. 2009) for the SB templates.

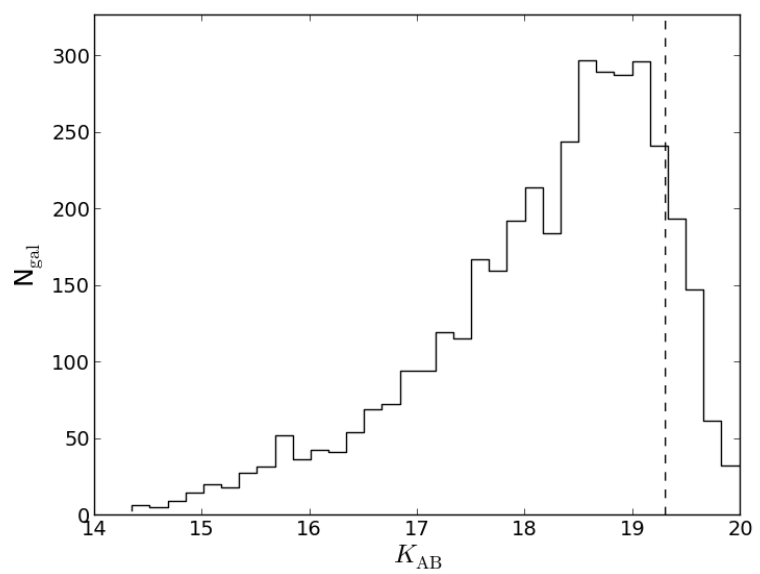

Fig. 2. $K_{\mathrm{AB}}$ magnitude distribution of the 3068 field galaxies identified in the sample. The steep drop-off after $K \sim 19.3$ is due to the criteria that $\sigma_{K} \leq 0.10$ for each galaxy, and that is it detected in five other bands. This represents a decline in completeness of the sample. The typical $3 \sigma$ limiting magnitude for an individual image as determined from the sky background is $K_{\mathrm{AB}} \sim 20.4$.

The values of extinction range from $0.0<E(B-V)<0.5$ in steps of 0.1 . Early type galaxy templates are not corrected for extinction, as there are no well-tested empirical models to describe the dust distribution for these galaxies. In the case of galaxy templates, redshift is constrained to $z<2$ with a step size $\Delta z=0.01$. The former constraint removes some degeneracy between high and low redshift template fits, and is justified since the population of galaxies with an apparent $K_{\mathrm{AB}}<20.0$ is negligibly small at $z \gtrsim 1.5$. We assume that the contamination from AGN-dominated sources in our galaxy catalog is negligible ( $\lesssim 5 \%$, as discussed later in the paper). We separate galaxies and stars based on their best fit template and shape parameters. Any sources with a full-width half maximum (FWHM) or ellipticity as measured with SExtractor in $5 \sigma$ excess of the stellar values for that field are automatically classified as galaxies. These stellar values are determined by computing the average and standard deviation of the lowest 20\% FWHM and ellipticity distributions, under the assumption that those "bottom $20 \%$ " of sources are stars. In practice, this usually means the FWHM and ellipticity of 5-10 stars are averaged for a given field. Besides this shape criterion, we categorize sources with $1.5 \times \chi_{\text {star }}^{2}>\chi_{\text {galaxy }}^{2}$ as galaxies, where $\chi^{2}$ corresponds to the best fit template for that class of source. This latter criterion has been applied to the COSMOS field with great success (Salvato et al. 2009). The extinction law and extinction values are to some extent free parameters in the fitting. Therefore, a certain amount of degeneracy in redshiftcolor-extinction space is expected. However, we do not expect this degeneracy to affect star/galaxy classification. This is due to the consideration of morphological information (i.e. extended vs point-like) and the implied redshift limit imposed by the $K$ band magnitude limit.

We detect 3068 galaxies and 1368 stars that match our criteria. The $K$-band magnitude distribution of galaxies is presented in Fig. 2.

\subsection{Verifying the source catalog}

To determine the accuracy of our galaxy-star separation, we compare a catalog of sources derived from nine GROND subfields of the COSMOS field with the COSMOS 30-band photometric catalog (COSMOS-30; Ilbert et al. 2009). COSMOS-30 

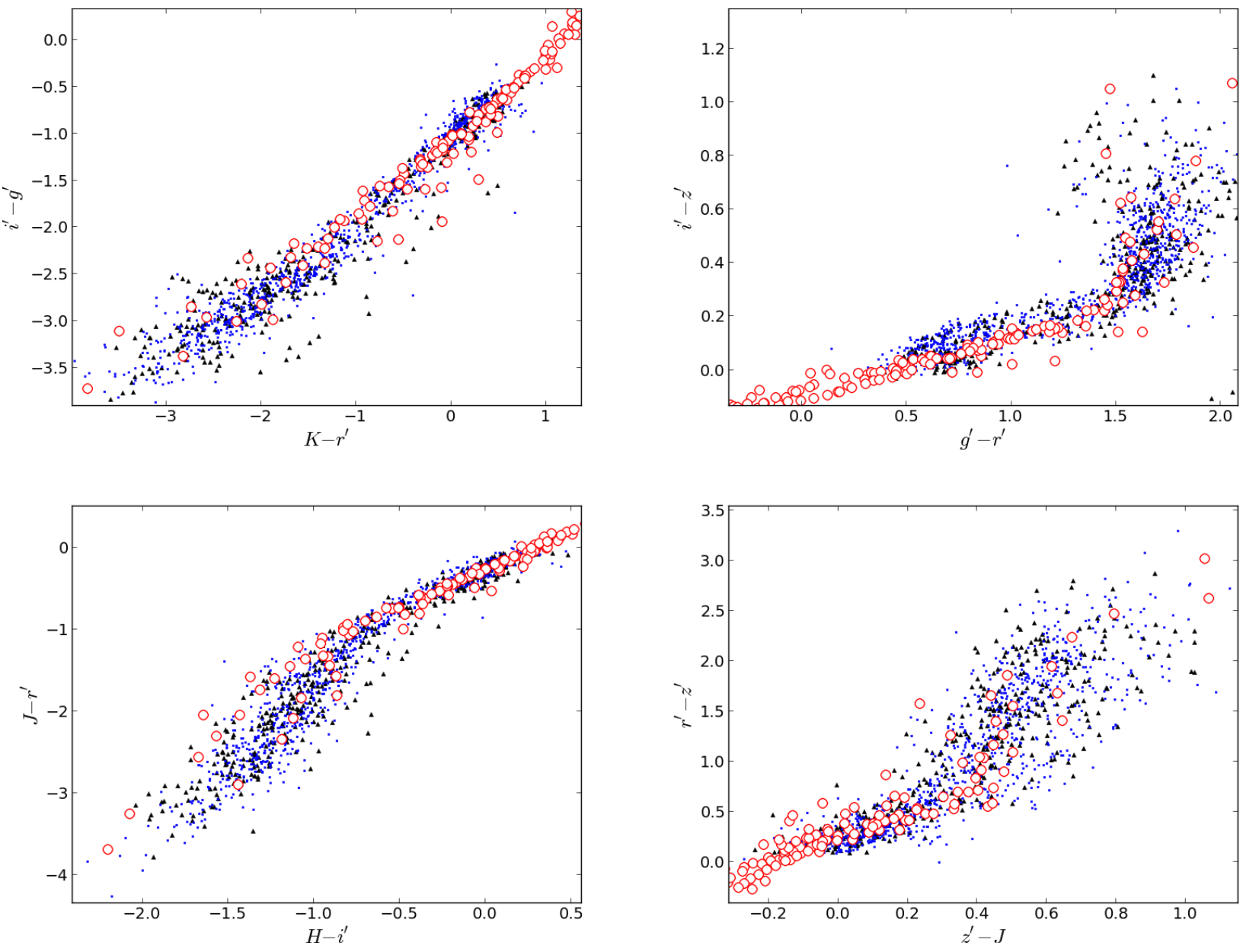

Fig. 3. Color-color diagrams for stellar templates (open circles) and sources identified as stars (black triangles and blue squares) in the GROND sample. Offsets of $+0.2,+0.08,+0.08$, and +0.1 mag to the $g^{\prime}, r^{\prime}, i^{\prime}$, and $z^{\prime}$ zeropoints are applied, except in the case of direct SDSS calibration. The sources represented by black triangles are SDSS calibrated, while the blue squares represent a zeropoint calibration.

has much larger spectral coverage and higher sensitivity than the GROND observations, making it an ideal standard with which to compare. We employ COSMOS-30 as both a measure of the quality of galaxy identification from GROND and to calculate the AGN-galaxy two point correlation function. From the nine adjacent sub-fields observed by GROND (center pointing at RA $=150.0862 \mathrm{deg}$, Dec $=2.3745 \mathrm{deg}$ ), we identify 317 total candidate galaxies. Nine of these candidates are in a masked area in COSMOS-30, and a further nine are categorized as stars in COSMOS-30. This corresponds to $2.9 \%$ of galaxies that are misclassified using our method, assuming COSMOS-30 has 100\% accuracy in classification. Besides the masked objects, no candidates were detected in GROND images without a corresponding source in COSMOS-30. The lack of spurious detections is likely a result from our stringent detection criteria discussed in Sect. 3.1 - namely, that an object must have independent detections in at least six filters.

We evaluate our photometric calibration by comparing the colors of sources that we classify as stars to the stellar templates of Pickles (1998) and Bohlin et al. (1995). In Fig. 3, we present color-color diagrams of our sources compared with those of stellar templates. On average, offsets of $+0.2,+0.08,+0.08$, and $+0.1 \mathrm{mag}$ to the $g^{\prime}, r^{\prime}, i^{\prime}$, and $z^{\prime}$ zeropoints, respectively, are required to match the colors of the stellar templates. In the case of direct SDSS calibration, no offset is needed nor applied. After applying these offsets, the colors of stars in our catalog are on average not offset from those of stellar templates, implying a reliable photometric calibration. The somewhat higher scatter of our objects relative to the templates is due to a combination of uncertain dust extinction toward foreground stars, binary systems, and photometric uncertainty.

\subsection{Measuring the two-point correlation}

After the source catalog is categorized, calculating a two-point GRB-galaxy correlation function is possible. Since our sample consists of isolated fields with no overlap, we must calculate distance pair distributions on a field by field basis, later combining them into a global correlation function. This technique limits the angular sizes we can examine, and furthermore introduces systematic errors when the size scales approaches the size of individual images. However, since we are specifically examining clustering at small scales, GROND's $5.4^{\prime} \times 5.4^{\prime}$ images $^{1}$ have sufficient spatial coverage. We limit our our analysis to angular distances $\leq 120^{\prime \prime}$. This angular distance corresponds to $\sim 600 \mathrm{kpc}$ at $z=0.4$, assuming a $\Lambda \mathrm{CDM}$ concordance cosmology with $h=0.7$ and $\Omega_{\mathrm{m}}=0.27$ (Wright 2006).

Many of the afterglows in our sample have no measured redshift. We assume that our sample of GRB afterglows has an underlying redshift distribution that is consistent with the literature (Jakobsson et al. 2012). More specifically, the GRBs should be background to the galaxies in the field. This latter assumption is

$15.4^{\prime} \times 5.4^{\prime}$ in $g^{\prime} r^{\prime} i^{\prime} z^{\prime}, 10.3^{\prime} \times 10.3^{\prime}$ in $J H K$. 


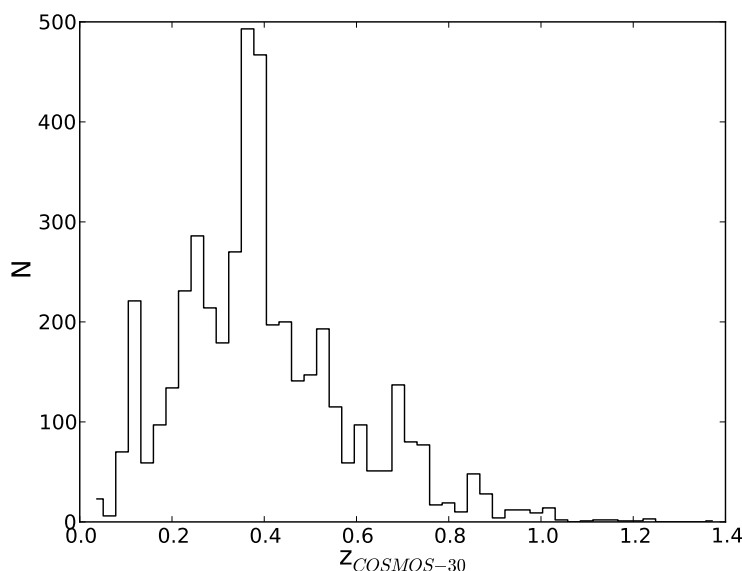

Fig. 4. Photometric redshift distribution of the 4481 galaxies in COSMOS-30 that have $K_{\mathrm{AB}}<19.3$. The mean and median redshifts of the distribution are 0.387 and 0.361 , respectively.

likely justified, since the mean redshift of GRBs is $z \sim 2$ and the mean redshift of galaxies with $K<19.3$ is $z \sim 0.4$, as noted in Sect. 2.

To measure the two-point correlation, we start by calculating the angular distance between the GRB optical afterglow positions (or the XRT afterglow position circle, in the case that no optical afterglow was detected) and each galaxy. This provides a distribution of arclengths $n_{\mathrm{DD}}$ for a given field. Next, we assign a new position to each galaxy within that field based on values picked from a uniform random distribution. Any galaxies that are within two stellar FWHM of a star or fall into an object mask are re-assigned a position until they do not. This ensures that the observability of the isotropically distributed control sample shares the same observational properties as the actual galaxies. We calculate the distances between each GRB-random galaxy $n_{\mathrm{DR}}$ and the random sample's mutual separation $n_{\mathrm{RR}}$. This process is repeated and averaged over a total of $10^{3}$ times for each field. We then calculate the angular correlation function $w$ and its variance $\sigma$ using the Landy and Szalay (Landy \& Szalay 1993) estimator

$w=\frac{\mathrm{DD}-2 \mathrm{DR}+\mathrm{RR}}{\mathrm{RR}}$,

$\sigma_{w}^{2}=\frac{1+w}{n_{\mathrm{DD}}}$

where DD, DR, and RR are the normalized frequency distributions of $n_{\mathrm{DD}}, n_{\mathrm{DR}}$, and $n_{\mathrm{RR}}$, respectively. We perform the same procedure to measure $w_{\text {galaxy-galaxy }}, w_{\mathrm{AGN}-\text { galaxy }}$, and

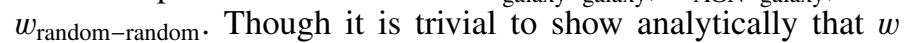
is zero in the case of isotropically distributed data, we explicitly perform the calculation using three randomly chosen coordinates per field as a verification of our method.

We compute the correlation functions on both the GROND and COSMOS-30 sample. To approximate the same selection criteria and completeness of our GROND sample, we remove from the COSMOS-30 catalog any sources with $K_{\mathrm{AB}}>19.3$ as well as those sources whose photometry may be unreliable due to saturation or being within an object mask (no flags in any optical filter). This leaves 4481 galaxies and 2273 stars. The photometric redshift distribution of these COSMOS-30 galaxies is presented in Fig. 4. The mean and median redshift of this sub-sample is 0.387 and 0.361 , respectively. We ensure that only background X-ray selected sources are included in the $w_{\text {AGN-galaxy }}$ calculation by imposing a $z>1.0$ redshift
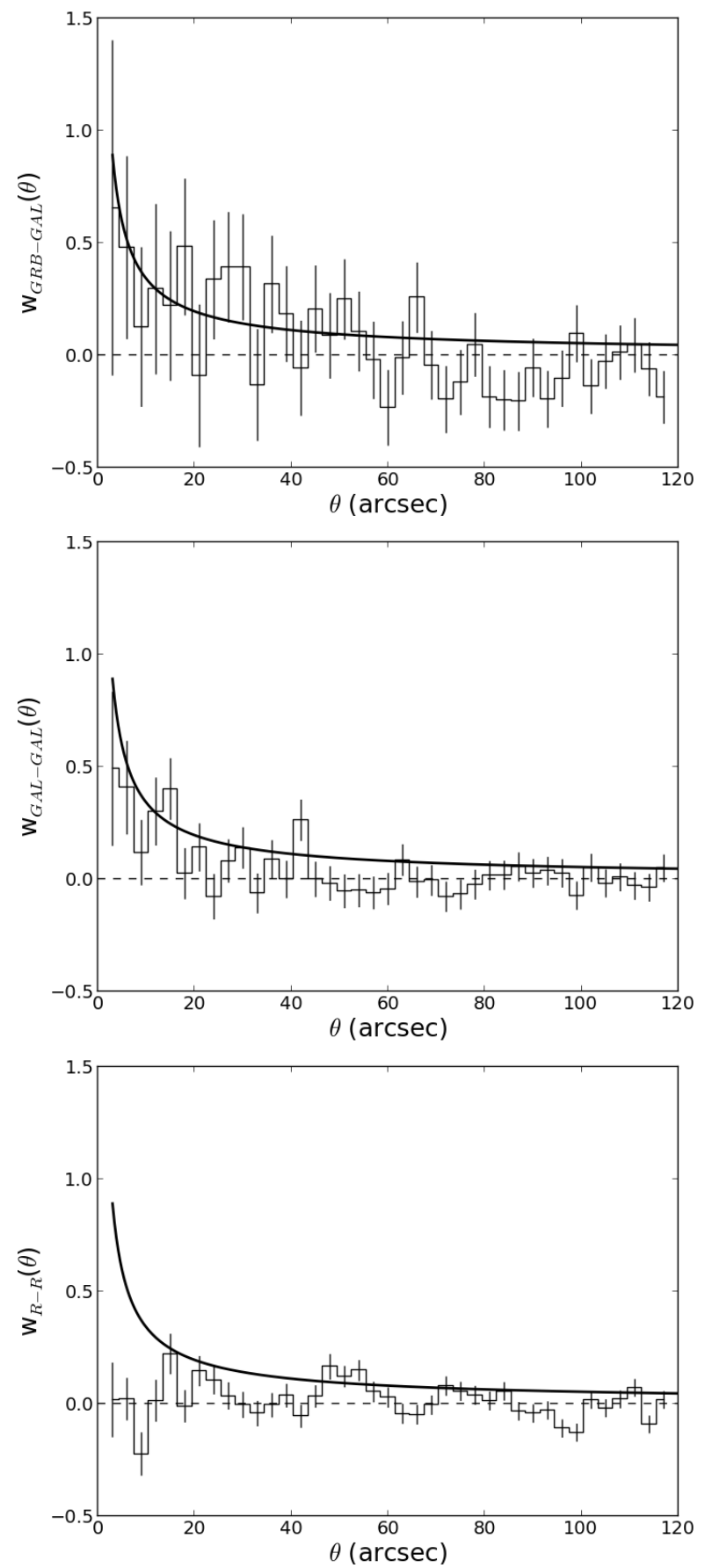

Fig. 5. (Top to bottom) GRB-galaxy, galaxy-galaxy, and randomrandom two point correlation functions. The solid black line represents the best fit power-law to the COSMOS-30 galaxy-galaxy two-point correlation function, as described in Fig. 6 and in Sect. 4. The randomrandom two-point correlation function is, as expected, consistent with zero.

criterion (Salvato et al. 2011). This criterion limits the catalog to 980 sources.

\section{Results}

In Fig. 5, we present the two point GRB-galaxy, galaxy-galaxy, and random-random angular correlation functions computed from the GRB sample. The two point correlation functions are self-consistent for all angular distance scales, with the exception of the (as expected) null random-random function. A possible 

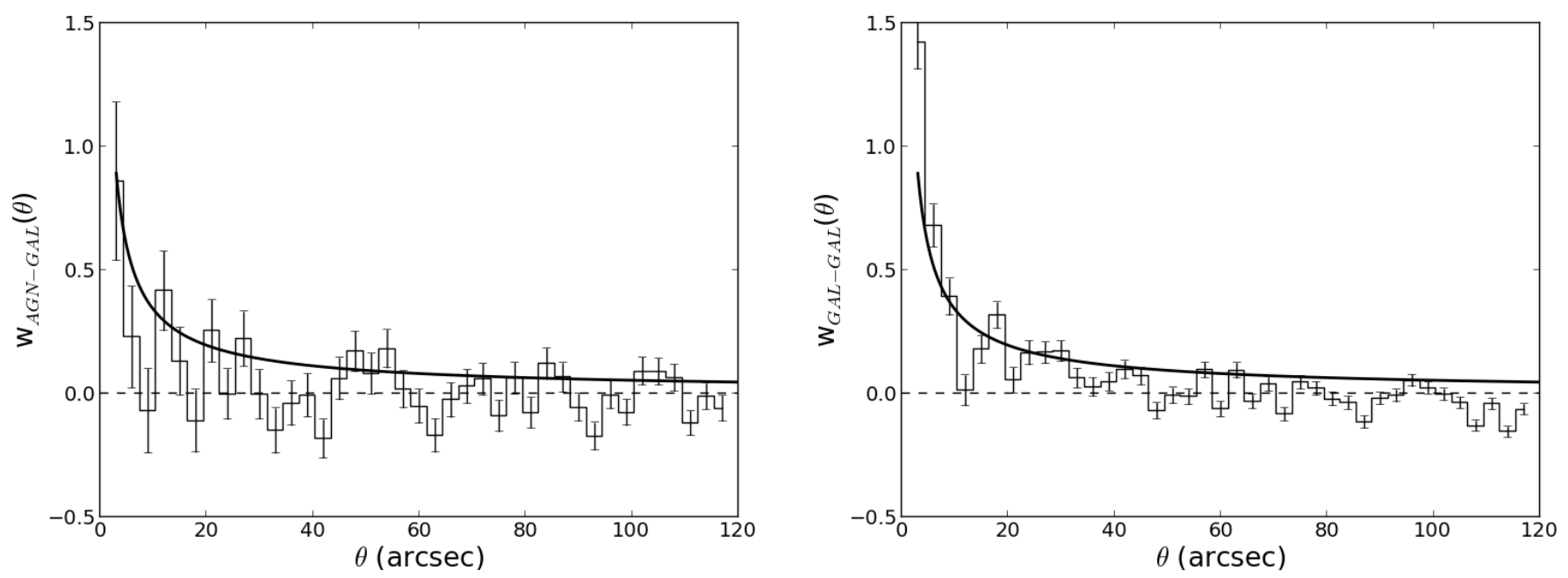

Fig. 6. (Left to right) AGN-galaxy and galaxy-galaxy two-point correlation functions computed from the COSMOS photometric catalog. To approximate the GROND sample selection and completeness, only sources with $K_{\mathrm{AB}}<19.3$ are included. The best fit power-law of the first 14 bins $\left(0 \leq \theta \leq 42^{\prime \prime}\right)$ of the galaxy-galaxy two-point correlation function is represented by the solid black line. The same fit is used in both figures for comparison.

contribution to the first bin of the GRB-galaxy $w_{\mathrm{GRB}-\mathrm{GAL}}$ is likely due to the detection of the host galaxy of the GRB, although the vast majority of known GRB hosts are fainter than our magnitude limit. This corresponds to $3^{\prime \prime}$ in our sample, and it is entirely possibly to find GRB host galaxies at up to these distances from the afterglow position and larger. Furthermore, these are also the angular distance scales within which Chen et al. (2009) find sources nearby sight-lines to GRBs with spectroscopically identified intervening MgII systems, albeit at very faint magnitudes.

We also find clustering of galaxies within small angular distances of each other as seen in the galaxy-galaxy correlation $w_{\mathrm{GAL}-\mathrm{GAL}}$. This is consistent with expectations from multiple galaxies occupying the same massive halo (Berlind \& Weinberg 2002; Lee et al. 2006) and naturally from galaxy clusters. This signal is seen more clearly in the correlation function as measured from the COSMOS-30 sample (Fig. 6), where we include only sources with $K_{\mathrm{AB}} \leq 19.3$ to approximate our sample's completeness. For the COSMOS-30 sample, the best fit power-law to the first 14 bins $\left(0 \leq \theta \leq 42^{\prime \prime}\right)$ of the form $\left(\theta / \theta_{0}\right)^{-\delta}$ yields a correlation length $\theta_{0}=2.6 \pm 0.4$ arcsec and slope $\delta=0.8 \pm 0.2$ (statistical error only). This same power-law is presented in all two-point correlation functions for comparison.

The AGN-galaxy two-point correlation function $w_{\mathrm{AGN}-\mathrm{GAL}}$ is also presented in Fig. 5.

\section{Discussion}

A $w_{\mathrm{GRB}-\mathrm{gal}}$ that is consistent with $w_{\mathrm{GAL}-\mathrm{GAL}}$ and $w_{\mathrm{AGN}-\mathrm{GAL}}$ implies that there is no excess or unusual clustering of galaxies around GRB events, contrary to the expectation from observations of MgII absorption line systems. Though our data support this null hypothesis, one must consider that the limiting magnitude of our sample is relatively shallow in comparison to the expected magnitudes of galaxies hosting MgII absorbers at the redshifts found in GRB afterglow spectra. Indeed, the typical magnitudes of candidate absorption line counterparts detected by Schulze et al. (2012) have $K_{\mathrm{AB}}>22$, and Chen et al. (2009) find that the objects at angular distances of sources $1^{\prime \prime}-3^{\prime \prime}$ to GRB hosts with spectroscopically detected MgII typically have $H_{\mathrm{AB}} \gtrsim 26$.
Although we expect that an overdensity of MgII absorbers corresponds to an overdensity of galaxies around the line of sight, we are unfortunately limited to the very brightest and closest objects in our survey. A qualitative measure of how much low number statistics affect $w$ is demonstrated by comparing our galaxy-galaxy correlation with that calculated from COSMOS-30 (Figs. 5, 6). The correlation as measured by 3068 galaxies in GROND fields only shows a hint of clustering due to halo occupation of multiple galaxies and galaxy clusters, as discussed in Sect. 4. The signal is much more clear from the 4481 galaxies with $K_{\mathrm{AB}}<19.3$ that we extract from COSMOS-30.

Clustering analysis of GRB afterglows and/or hosts have been performed by Wang \& Wei (2010); Bornancini et al. (2004). Wang \& Wei (2010) find no evidence of clustering between Swift XRT afterglows and ROSAT selected galaxy clusters. The purpose of our survey is not to examine large scale anisotropies, but rather the small scale clustering implied by the MgII excess. Bornancini et al. (2004) examined whether GRB host galaxies tend to reside in high density environments by calculating the GRB-galaxy two point correlation for 19 GRB hosts. They concluded that GRB host galaxies likely do not occur in over-dense areas, although local cosmic variance could still significantly affect these results due to the small sample size. Furthermore, choosing hosts over afterglows introduces an additional bias against intrinsically dim or reddened hosts. Constructing a sample based on afterglow positions - as done in this work - removes this bias and increases the sample size significantly. Furtheremore, there is no a priori reason to link foreground absorption in the afterglow with the detection or non-detection of a host galaxy.

The largest limitation to our survey stems from the fact that there is significant degeneracy between galactic/galactic and galactic/stellar templates in GROND's optical regime for objects at $z<2$. For this reason, including NIR data is crucial to distinguish point-like galaxies from stars. The sensitivity of GROND's $K$ channel is typically three magnitudes shallower than its optical channels. Thus, we are forced to exclude a large number of sources from our survey simply due to insufficient sensitivity. Including Spitzer/IRAC or HST/WFC3 data for a significant fraction of fields observed by GROND would remove the largest 
constraint to our survey by providing much deeper sensitivity limits and/or wavelength coverage, simultaneously increasing statistics and enabling the investigation of a much larger redshift space. Such surveys are currently underway and are scheduled to be completed in the next years (Levan 2009b,a; Perley et al. 2012). Combining these high quality NIR data with GROND data would also provide for accurate photometric redshifts, enabling a measure of the two point spatial correlation function.

Acknowledgements. We thank the anonymous referee for his or her helpful comments. We thank Sotoria Fotopoulou for insightful discussion regarding photometric calibration techniques, and David Gruber for his projection plotting routine. S.K., D.A.K., and A.R. acknowledge support by DFG grant K1 766/16-1. S.S. acknowledges support through project M.FE.A.Ext 00003 of the MPG, and P.S. acknowledges support by DFG grant SA 2001/1-1. T.K. acknowledges support by the European Commission under the Marie Curie Intra-European Fellowship Programme. The Dark Cosmology Centre is funded by the Danish National Research Foundation.

\section{References}

Arnouts, S., Cristiani, S., Moscardini, L., et al. 1999, MNRAS, 310, 540 Basa, S., Cuby, J. G., Savaglio, S., et al. 2012, A\&A, 542, A103

Berlind, A. A., \& Weinberg, D. H. 2002, ApJ, 575, 587

Bertin, E., \& Arnouts, S. 1996, A\&AS, 117, 393

Bohlin, R. C., Colina, L., \& Finley, D. S. 1995, AJ, 110, 1316

Bordoloi, R., Lilly, S. J., Knobel, C., et al. 2011, ApJ, 743, 10

Bordoloi, R., Lilly, S. J., Amara, A., et al. 2012, MNRAS, 421, 1671

Bornancini, C. G., Martínez, H. J., Lambas, D. G., et al. 2004, ApJ, 614, 84

Bouché, N., Murphy, M. T., Péroux, C., Csabai, I., \& Wild, V. 2006, MNRAS, 371,495

Brainerd, J. J., Meegan, C. A., Briggs, M. S., Pendleton, G. N., \& Brock, M. N. 1995, ApJ, 441, L39

Butler, N. R., Bloom, J. S., \& Poznanski, D. 2010, ApJ, 711, 495

Calzetti, D., Kinney, A. L., \& Storchi-Bergmann, T. 1994, ApJ, 429, 582

Chen, H.-W., Perley, D. A., Pollack, L. K., et al. 2009, ApJ, 691, 152

Churchill, C. W., Mellon, R. R., Charlton, J. C., et al. 2000, ApJ, 543, 577

Cucchiara, A., Jones, T., Charlton, J. C., et al. 2009, ApJ, 697, 345

Cucchiara, A., Prochaska, J. X., Zhu, G., et al. 2012, ApJ, submitted [arXiv: 1211.6528]

Elliott, J., Greiner, J., Khochfar, S., et al. 2012, A\&A, 539, A113

Frank, S., Bentz, M. C., Stanek, K. Z., et al. 2007, Ap\&SS, 312, 325

Greiner, J., Bornemann, W., Clemens, C., et al. 2008, PASP, 120, 405

Greiner, J., Krühler, T., Klose, S., et al. 2011, A\&A, 526, A30
Groth, E. J., \& Peebles, P. J. E. 1977, ApJ, 217, 385

Ilbert, O., Arnouts, S., McCracken, H. J., et al. 2006, A\&A, 457, 841

Ilbert, O., Capak, P., Salvato, M., et al. 2009, ApJ, 690, 1236

Jakobsson, P., Björnsson, G., Fynbo, J. P. U., et al. 2005, MNRAS, 362, 245

Jakobsson, P., Hjorth, J., Malesani, D., et al. 2012, ApJ, 752, 62

Kacprzak, G. G., \& Churchill, C. W. 2011, ApJ, 743, L34

Kann, D. A., Klose, S., Zhang, B., et al. 2010, ApJ, 720, 1513

Krühler, T., Greiner, J., Schady, P., et al. 2011, A\&A, 534, A108

Landy, S. D., \& Szalay, A. S. 1993, ApJ, 412, 64

Le Floc'h, E., Charmandaris, V., Forrest, W. J., et al. 2006, ApJ, 642, 636

Lee, K.-S., Giavalisco, M., Gnedin, O. Y., et al. 2006, ApJ, 642, 63

Levan, A. 2009a, in HST Proposal, 12307

Levan, A. 2009b, in HST Proposal, 12378

López, G., \& Chen, H.-W. 2012, MNRAS, 419, 3553

Lopez, S., Barrientos, L. F., Lira, P., et al. 2008, ApJ, 679, 1144

Matejek, M. S., \& Simcoe, R. A. 2012, ApJ, 761, 112

Matejek, M. S., Simcoe, R. A., Cooksey, K. L., \& Seyffert, E. N. 2013, ApJ, 764, 9

Peebles, P. J. E. 1980, The large-scale structure of the universe

Perley, D., Tanvir, N., Hjorth, J., et al. 2012, Spitzer Proposal, 90062

Perley, D. A., Levan, A. J., Tanvir, N. R., et al. 2013, ApJ, submitted [arXiv: 1301.5903]

Pickles, A. J. 1998, PASP, 110, 863

Porciani, C., Viel, M., \& Lilly, S. J. 2007, ApJ, 659, 218

Prevot, M. L., Lequeux, J., Prevot, L., Maurice, E., \& Rocca-Volmerange, B. 1984, A\&A, 132, 389

Prochaska, J. X., Chen, H.-W., \& Bloom, J. S. 2006, ApJ, 648, 95

Prochter, G. E., Prochaska, J. X., Chen, H.-W., et al. 2006, ApJ, 648, L93

Rapoport, S., Onken, C. A., Schmidt, B. P., et al. 2012, ApJ, 754, 139

Ross, N. P., Shen, Y., Strauss, M. A., et al. 2009, ApJ, 697, 1634

Salvaterra, R., Campana, S., Vergani, S. D., et al. 2012, ApJ, 749, 68

Salvato, M., Hasinger, G., Ilbert, O., et al. 2009, ApJ, 690, 1250

Salvato, M., Ilbert, O., Hasinger, G., et al. 2011, ApJ, 742, 61

Savaglio, S., Glazebrook, K., \& Le Borgne, D. 2009, ApJ, 691, 182

Schulze, S., Fynbo, J. P. U., Milvang-Jensen, B., et al. 2012, A\&A, 546, A20

Steidel, C. C., Pettini, M., \& Hamilton, D. 1995, AJ, 110, 2519

Sudilovsky, V., Savaglio, S., Vreeswijk, P., et al. 2007, ApJ, 669, 741

Sudilovsky, V., Smith, D., \& Savaglio, S. 2009, ApJ, 699, 56

Tanvir, N. R., Levan, A. J., Fruchter, A. S., et al. 2012, ApJ, 754, 46

Tejos, N., Lopez, S., Prochaska, J. X., Chen, H.-W., \& Dessauges-Zavadsky, M. 2007, ApJ, 671, 622

Vergani, S. D., Petitjean, P., Ledoux, C., et al. 2009, A\&A, 503, 771

Wang, J., \& Wei, J.-Y. 2010, Res. Astron. Astrophys., 10, 533

Wright, E. L. 2006, PASP, 118, 1711

Wyithe, J. S. B., Oh, S. P., \& Pindor, B. 2011, MNRAS, 414, 209

Zhu, G., \& Ménard, B. 2012, ApJ, submitted [arXiv: 1211.6215] 
Table 1. Identification and positions of the GRBs in the final sample.

\begin{tabular}{|c|c|c|}
\hline GRB & RA J2000 & Dec J2000 \\
\hline 071112C & 02:36:50.93 & $+28: 22: 16.68$ \\
\hline 080212 & $15: 24: 35.42$ & $-22: 44: 29.70$ \\
\hline 080330 & $11: 17: 04.51$ & $+30: 37: 23.48$ \\
\hline 080408 & 07:38:39.59 & $+33: 18: 14.90$ \\
\hline 080413B & $21: 44: 34.67$ & $-19: 58: 52.40$ \\
\hline 080413 & 19:09:11.74 & $-27: 40: 40.30$ \\
\hline 080514B & $21: 31: 22.69$ & $+00: 42: 28.90$ \\
\hline 080520 & $18: 40: 46.30$ & $-54: 59: 31.00$ \\
\hline 080523 & 01:23:11.70 & $-64: 01: 51.50$ \\
\hline 080605 & $17: 28: 30.05$ & $+04: 00: 55.97$ \\
\hline 080710 & 00:33:05.67 & $+19: 30: 05.69$ \\
\hline 080916 & $22: 25: 06.20$ & $-57: 01: 22.90$ \\
\hline 080928 & $06: 20: 16.82$ & $-55: 11: 59.30$ \\
\hline 081008 & $18: 39: 49.89$ & $-57: 25: 52.80$ \\
\hline 081109 & 22:03:09.86 & $-54: 42: 41.04$ \\
\hline 081118 & $05: 30: 22.18$ & $-43: 18: 05.30$ \\
\hline 081121 & 05:57:06.08 & $-60: 36: 09.94$ \\
\hline 081228 & 02:37:50.89 & $+30: 51: 09.10$ \\
\hline 081221 & 01:03:10.19 & $-24: 32: 52.20$ \\
\hline 081230 & $02: 29: 19.53$ & $-25: 08: 51.72$ \\
\hline 090102 & 08:32:38.10 & $+33: 11: 45.30$ \\
\hline 090123 & 00:27:08.74 & $-23: 30: 04.00$ \\
\hline 090205 & $14: 43: 38.65$ & $-27: 51: 10.70$ \\
\hline 090323 & $12: 42: 50.29$ & $+17: 03: 11.60$ \\
\hline 090401B & $06: 20: 21.10$ & $-08: 58: 19.35$ \\
\hline 090423 & 09:55:33.29 & $+18: 08: 58.00$ \\
\hline 090424 & 12:38:05.09 & $+16: 50: 14.75$ \\
\hline 090426 & $12: 36: 18.04$ & $+32: 59: 09.24$ \\
\hline 090509 & 16:05:39.01 & $-28: 23: 59.64$ \\
\hline 090516 & 09:13:02.59 & $-11: 51: 14.90$ \\
\hline 090519 & 09:29:07.00 & $+00: 10: 49.10$ \\
\hline 090530 & 11:57:40.51 & $+26: 35: 38.40$ \\
\hline 090812 & $23: 32: 48.54$ & $-10: 36: 17.60$ \\
\hline 090814 & $15: 58: 26.35$ & $+25: 37: 52.42$ \\
\hline 090902B & $17: 39: 45.41$ & $+27: 19: 27.10$ \\
\hline 090926B & 03:05:13.94 & $-39: 00: 22.20$ \\
\hline 091018 & 02:08:44.61 & $-57: 32: 53.70$ \\
\hline 091029 & 04:00:42.60 & $-55: 57: 19.84$ \\
\hline 091109 & 20:37:01.80 & $-44: 09: 29.60$ \\
\hline 100219A & $10: 16: 48.50$ & $-12: 34: 00.50$ \\
\hline $100414 \mathrm{~A}$ & $12: 48: 26.93$ & $+08: 41: 34.40$ \\
\hline $100518 \mathrm{~A}$ & 20:19:09.33 & $-24: 33: 16.56$ \\
\hline $100621 \mathrm{~A}$ & 21:01:13.11 & $-51: 06: 22.46$ \\
\hline $100902 \mathrm{~A}$ & 03:14:30.96 & $+30: 58: 45.23$ \\
\hline $101023 \mathrm{~A}$ & $21: 11: 51.23$ & $-65: 23: 15.61$ \\
\hline 101219B & $00: 48: 55.34$ & $-34: 33: 59.26$ \\
\hline $110128 \mathrm{~A}$ & $12: 55: 35.10$ & $+28: 03: 54.10$ \\
\hline $110206 \mathrm{~A}$ & 06:09:20.04 & $-58: 48: 24.91$ \\
\hline $110312 \mathrm{~A}$ & 10:29:55.47 & $-05: 15: 45.20$ \\
\hline $110407 \mathrm{~A}$ & $12: 24: 07.49$ & $+15: 42: 42.16$ \\
\hline 110709B & 10:58:37.11 & $-23: 27: 16.76$ \\
\hline
\end{tabular}

Table 1. continued.

\begin{tabular}{lcc}
\hline \hline GRB & RA J2000 & Dec J2000 \\
\hline 110721A & $22: 14: 38.19$ & $-38: 35: 35.70$ \\
110818A & $21: 09: 20.89$ & $-63: 58: 51.80$ \\
111008A & $04: 01: 48.22$ & $-32: 42: 34.09$ \\
111107A & $08: 37: 54.65$ & $-66: 31: 12.40$ \\
111129A & $20: 29: 44.14$ & $-52: 42: 46.48$ \\
111209A & $00: 57: 22.70$ & $-46: 48: 05.00$ \\
111211A & $10: 12: 21.70$ & $+11: 12: 30.00$ \\
111212A & $20: 41: 43.52$ & $-68: 36: 45.00$ \\
111228A & $10: 00: 16.01$ & $+18: 17: 51.80$ \\
111229A & $05: 05: 08.84$ & $-84: 42: 38.70$ \\
120119A & $08: 00: 06.94$ & $-09: 04: 53.83$ \\
120211A & $05: 51: 00.89$ & $-24: 46: 30.79$ \\
120302A & $08: 09: 35.54$ & $+29: 37: 41.05$ \\
120311A & $18: 12: 22.16$ & $+14: 17: 46.30$ \\
120320A & $14: 10: 04.30$ & $+08: 41: 47.26$ \\
120404A & $15: 40: 02.29$ & $+12: 53: 06.29$ \\
120422A & $09: 07: 38.42$ & $+14: 01: 07.36$ \\
121024A & $04: 41: 53.30$ & $-12: 17: 26.48$ \\
121027A & $04: 14: 23.45$ & $-58: 49: 47.17$ \\
121217A & $10: 14: 50.51$ & $-62: 21: 03.28$ \\
121229A & $12: 40: 24.29$ & $-50: 35: 39.48$ \\
\hline & & \\
\hline
\end{tabular}

